Pacific

Journal of

Mathematics

TEST WORDS, GENERIC ELEMENTS AND ALMOST PRIMITIVITY

B. Fine, G. Rosenberger, D. Spellman, and M. Stille 


\title{
TEST WORDS, GENERIC ELEMENTS AND ALMOST PRIMITIVITY
}

\author{
B. Fine, G. Rosenberger, D. Spellman, and M. Stille
}

\begin{abstract}
A test element in a group $G$ is an element $g$ with the property that if $f(g)=g$ for an endomorphism $f$ of $G$ to $G$ then $f$ must be an automorphism. A test element in a free group is called a test word. Nielsen gave the first example of a test word by showing that in the free group on $x, y$ the commutator $[x, y]$ satisfies this property. T. Turner recently characterized test words as those elements of a free group contained in no proper retract. Since free factors are retracts, test words are therefore very strong forms of non-primitive elements. In this paper we give some new examples of test words and examine the relationship between test elements and several other concepts, in particular generic elements and almost-primitive elements (APE's). In particular we show that an almost primitive element which lies in a certain type of verbal subgroup must be a test word. Further using a theorem of Rosenberger on equations in free products we prove a result on APE's, generic elements and test words in certain free products of free groups. Finally we examine test elements in non-free groups and introduce the concept of the test rank of a group.
\end{abstract}

\section{Introduction.}

A test element in a group $G$ is an element $g$ with the property that if $f(g)=g$ for an endomorphism $f$ of $G$ to $G$ then $f$ must be an automorphism. A test element in a free group is called a test word. Nielsen $[\mathbf{N}]$ gave the first non-trivial example of a test word by showing that in the free group on $x, y$ the commutator $[x, y]$ satisfies this property. T. Turner $[\mathbf{T}]$ recently characterized test words as those elements of a free group which do not lie in any proper retract. Using this characterization he was able to give several straightforward criteria to determine if a given element of a free group is a test word. Using these criteria, Comerford $[\mathbf{C}]$ proved that it is effectively decidable whether elements of free groups are test words. Since free factors are retracts, Turner's result implies that no test word can fall in a proper free factor. Therefore being a test word is a very strong form of non-primitivity.

In this paper we consider relationships between test words and two related concepts - almost primitive elements (APE's) and generic elements. 
We give the formal definitions in the next section where we also prove that an almost primitive element of a free group which lies in a certain type of verbal subgroup must be a test word (Theorem 1). This is quite surprising given the strong non-primitivity of test words. In Section 3 we use a theorem of Rosenberger $[\mathbf{R} 1]$ on equations in free products to prove a result on APE's, generic elements and test words in certain free products of free groups. In Section 4, using Nielsen transformations, we produce a set of generic elements in the free group of rank two. Using the theorem of Rosenberger mentioned above, these examples can be extended to finding generic elements in higher rank free groups. Finally in Section 5 we give some straightforward results on extensions of these concepts to arbitrary non-free groups. As pointed out by Turner the characterization of test elements in general is more subtle and difficult than in the free group case.

We note that a few of the results appear in the Diplomarbeit of N. Isermann $[\mathbf{I}]$ however the proofs given here are somewhat different.

\section{Test Words, Almost Primitive Elements and Generic Elements.}

A test element in a group $G$ is an element $g$ with the property that if $f(g)=g$ for an endomorphism $f$ of $G$ to $G$ then $f$ must be an automorphism. A test element in a free group is called a test word. Nielsen $[\mathbf{N}]$ gave the first non-trivial example of a test word by showing that in the free group on $x, y$ the commutator $[x, y]$ satisfies this property. Other examples of test words have been given by Zieschang $[\mathbf{Z 1}, \mathbf{Z 2}]$, Rosenberger $[\mathbf{R 1}, \mathbf{R 2}, \mathbf{R 3}]$ Kalia and Rosenberger $[\mathbf{K}-\mathbf{R}]$, Hill and Pride $[\mathbf{H}-\mathbf{P}]$ and Durnev $[\mathbf{D}]$. Gupta and Shpilrain $[\mathbf{G}-\mathbf{S}]$ have studied the question as to whether the commutator $[x, y]$ is a test element in various quotients of the free group on $x, y$.

Recall that a subgroup $H$ of a group $G$ is a retract if there exists a homomorphism $f: G \rightarrow H$ which is the identity on $H$. Clearly in a free group $F$ any free factor is a retract. However there do exist retracts in free groups which are not free factors. Recently T. Turner $[\mathbf{T}]$ characterized test words as those elements of a free group which do not lie in any proper retract. Using this characterization he was able to give several straightforward criteria to determine if a given element of a free group is a test word. Using these criteria, Comerford $[\mathbf{C}]$ proved that it is effectively decidable whether elements of free groups are test words. Since free factors are retracts, Turner's result implies that no test word can fall in a proper free factor. Therefore being a test word is a very strong form of non-primitivity. Shpilrain $[\mathbf{S 1}, \mathbf{S 2}]$ defined the rank of an element $w$ in a free group $F$ as the smallest rank of a free factor containing $w$. Clearly in a free group of rank $n$ a test word has maximal rank $n$. Shpilrain conjectured that the converse was also true but Turner gave an example showing this to be false. However 
Turner also proved that Shpilrain's conjecture is true if only test words for monomorphisms are considered.

As a direct consequence of the characterization Turner obtains the following result [T, Example 5] which shows that there is a fairly extensive collection of test words in a free group of rank two.

Proposition $1([\mathbf{T}])$. In a free group of rank two any non-trivial element of the commutator subgroup is a test word.

Proof. Let $F$ be a free group of rank two and suppose $H$ is a proper retract. Then the rank of $H$ must be one and hence $H$ is abelian. Suppose $g \in F^{\prime}$ the commutator subgroup of $F$. If $g \in H$ then there exists an endomorphism $f: F \rightarrow H$ which is the identity on $H$. Therefore $f(g)=g$. But $f(g)=1$ if $f$ is any homomorphism of $F$ into an abelian group. Therefore $g=1$. It follows that no non-trivial element of $F^{\prime}$ can lie in any proper retract and therefore by Turner's characterization must be a test word.

An almost primitive element - (APE) - is an element of a free group $F$ which is not primitive in $F$ but which is primitive in any proper subgroup of $F$ containing it. This can be extended to arbitrary groups in the following manner. An element $g \in G$ is primitive in $G$ if $g$ generates an infinite cyclic free factor of $G$, that is $g$ has infinite order and $G=\langle g\rangle \star G_{1}$ for some $G_{1} \subset G . g$ is then an APE if it is not primitive in $G$ but primitive in any proper subgroup containing it. Rosenberger $[\mathbf{R} 1]$ proved that in the free group $F=F\left(x_{i}, y_{i}, z_{j}\right) ; 1 \leq i \leq m, 1 \leq j \leq n$, of rank $2 m+n$ the element

$$
\left[x_{1}, y_{1}\right] \ldots\left[x_{m}, y_{m}\right] z_{1}^{p_{1}} \ldots z_{n}^{p_{n}}
$$

where the $p_{i}$ are not necessarily distinct primes, is an APE in $F$. Rosenberger [R1] proved, in a different setting that if $A, B$ are arbitrary groups containing APE's $a, b$ respectively, then the product $a b$ is either primitive or an APE in the free product $A \star B$. This was reproved by Brunner, Burns and Oates-Williams [B-B-O] who also prove the more difficult result that if $a$ and $b$ are tame APE's in groups $A, B$ respectively then their product normally is a tame APE in $A \star B$. An APE $w$ in a group $G$ ia a tame APE if whenever $w^{\alpha} \in H \subset G$ with $\alpha \geq 1$ minimal, then either $w^{\alpha}$ is primitive in $H$ or the index $[G: H]$ is $\alpha$. It follows easily that $\left[a_{1}, b_{1}\right] \ldots\left[a_{g}, b_{g}\right], g \geq 1$, is a tame APE in the free group on $a_{1}, b_{1} \ldots a_{g}, b_{g}$, (see $[\mathbf{R} 3]$ ). We note that Brunner, Burns and Oates-Williams give a more technical definition of a tame APE.

Let $\mathcal{U}$ be a variety defined by a set of laws $\mathcal{V}$. (We refer to the book of $\mathrm{H}$. Neumann $[\mathrm{Ne}]$ for relevant terminology.) For a group $G$ we let $\mathcal{V}(G)$ denote the verbal subgroup of $G$ defined by $\mathcal{V}$. An element $g \in G$ is $\mathcal{U}$-generic in $G$ if $g \in \mathcal{V}(G)$ and whenever $H$ is a group, $f: H \rightarrow G$ a homomorphism and $w=f(u)$ for some $u \in \mathcal{V}(H)$ it follows that $f$ is surjective. Equivalently $g \in G$ is $\mathcal{U}$-generic in $G$ if $g \in \mathcal{V}(G) \subset G$ but $g \notin \mathcal{V}(K)$ for every proper 
subgroup $K$ of $G$ [St]. An element is generic if it is $\mathcal{U}$-generic for some variety $\mathcal{U}$. Let $\mathcal{U}_{n}$ be the variety defined by the set of laws $\mathcal{V}_{n}=\left\{[x, y], z^{n}\right\}$. For $n=0$ we have $\mathcal{U}_{n}=\mathcal{A}$ the abelian variety. Stallings [St] and Dold [Do] have given sufficient conditions for an element of a free group to be $\mathcal{U}_{n}$-generic. Using this it can be shown that $x_{1}^{n} x_{2}^{n} \ldots x_{m}^{n}$ is $\mathcal{U}_{n}$-generic in the free group on $x_{1}, \ldots, x_{m}$ for all $n \geq 2$ and if $m$ is even $\left[x_{1}, x_{2}\right], \ldots\left[x_{m-1}, x_{m}\right]$ is $\mathcal{U}_{n}$-generic in the free group on $x_{1}, \ldots, x_{m}$ for $n=0$ and for all $n \geq 2$. These facts are also consequences of a result of Rosenberger [R2, R3].

Comerford $[\mathbf{C}]$ points out that if $G$ is Hopfian, which is the case if $G$ is free, then being generic implies being a test word. Thus for free groups we have

\section{generic $\longrightarrow$ test word .}

Comerford also shows that there is no converse. In particular he shows that in a free group of rank 3 on $x, y, z$ the word $w=x^{2}\left[y^{2}, z\right]$ is a test word but is not generic. We can also show that in general, generic does not imply APE. Suppose $F=F(x, y)$ is the free group of rank two on $x, y$ and let $w=x^{4} y^{4}$. Then $w$ is $\mathcal{U}_{4}$-generic but $w$ is not an APE since $w \in\left\langle x^{2}, y^{2}\right\rangle$ and is not primitive in this subgroup while this subgroup is not all of $F$.

Further, in general it is not true that being an APE implies being a test word. Again let $F=F(x, y)$ and let $w=x^{2} y x^{-1} y^{-1}$. Brunner, Burns and Oates-Williams, after a private communication with G. Rosenberger, show that $w$ is an APE. However Turner shows that $w$ is not a test word. Since generic elements are test words in a Hopfian group this example shows further that APE does not imply generic in general. This is really to be expected since test words are strongly non-primitive. However our first result shows that many APE's are indeed generic and therefore test words.

Recall that a variety $\mathcal{U}$ defined by the set of laws $\mathcal{V}$ is a non-trivial variety if it consists of more than just the trivial group. In this case $\mathcal{V}(F) \neq F$ for any free group $F$.

Theorem 1. Let $F$ be a free group and $\mathcal{B}$ a non-trivial variety defined by the set of laws $\mathcal{V}$. Let $w \in \mathcal{V}(F)$. If $w$ is an APE then $w$ is $\mathcal{B}$-generic. In particular $w$ is a test word.

Proof. Let $w \in \mathcal{V}(F)$ be an APE and let $\phi: H \rightarrow F$ be a homomorphism with $\phi(u)=w$ for some $u \in \mathcal{V}(H)$. As in the statement of the theorem, $\mathcal{V}$ is the set of laws defining the non-trivial variety $\mathcal{B}$. Let $K$ be a proper subgroup of $F$. If $w \notin K$ then clearly $w \notin \mathcal{V}(K)$. If $w \in K$ then since $w$ is an APE, $w$ is primitive in $K$ since $K$ is a proper subgroup of $F$. Further since $\mathcal{B}$ is a non-trivial variety and $K$ is free we have that $K \neq \mathcal{V}(K)$. It follows then from the primitivity of $w$ in $K$ that $w \notin \mathcal{V}(K)$. Therefore $w \in \mathcal{V}(F)$ and for any proper subgroup $K$ of $F$ we have $w \notin \mathcal{V}(K)$ and hence $w$ is $\mathcal{B}$-generic. Since free groups are Hopfian, $w$ must then be a test word. 
In particular let $F(n)$ be the subgroup of the free group $F$ generated by all commutators and $n$-th powers, $n \geq 2$ or $n=0$, that is $F(n)=\mathcal{V}_{n}(F)$. Then:

Corollary 1. Let $w \in F(n)$ with $n \geq 2$ or $n=0$. If $w$ is an APE then $w$ is $\mathcal{U}_{n}$-generic and $w$ is a test word.

\section{APE's in Certain Free Products of Free Groups.}

In this section we give a result on APE's, generic elements and test words on certain free products of free groups. The result depends on the following theorem of Rosenberger [R1].

Theorem $2([\mathbf{R} 1])$. Let $G=H_{1} \star \cdots H_{n}, n \geq 2$, be the free product of groups $H_{1}, \ldots, H_{n}$. Let $a_{j} \in H_{j}, a_{j} \neq 1$, and let $p$ be the number of $a_{j}$ which are proper powers in $H_{j},(1 \leq j \leq n)$. Let $\left\{x_{1}, \ldots, x_{m}\right\} \subset G, m \geq 1$, and let $H$ be the subgroup of $G$ generated by $x_{1}, \ldots, x_{m}$. If $a=a_{1} \ldots a_{n} \in H$ then one of the following cases holds:

(1) There is a Nielsen transformation from $\left\{x_{1}, \ldots, x_{m}\right\}$ to a system $\left\{y_{1}\right.$, $\left.\ldots, y_{m}\right\}$ with $y_{1}=a_{1} \cdots a_{n}$.

(2) It is $m \geq 2 n-p$, and there is a Nielsen transformation from $\left\{x_{1}, \ldots\right.$, $\left.x_{m}\right\}$ to a system $\left\{y_{1}, \ldots, y_{m}\right\}$ with $y_{i} \in H_{j}, 1 \leq j \leq n, 1 \leq i \leq 2 n-p$; and moreover $a_{j}$ can be written as a word in those $y_{k}, 1 \leq k \leq m$, which are contained in $H_{j}, 1 \leq j \leq n$.

Proof. Here we give a more detailed proof than in $[\mathbf{R} \mathbf{1}]$. This is done in order to explain more extensively the concept of semistable letters and the blockwise description of letters in a product of generators.

We regard $G$ as the free product $G=H_{1} \star \cdots \star H_{n}$ together with the length $L$ and an order with respect to this factorization. We refer to the papers $[\mathbf{Z 3}]$ and $[\mathbf{F}-\mathbf{R}-\mathbf{S}]$ for the terminology and properties related to the length $L$ and Nielsen cancellation methods in such free products. Consider the sets of elements $\left\{x_{1}, \ldots, x_{m}\right\}$ and $\left\{a_{1}, \ldots, a_{n}\right\}$ as in the statement of the theorem. We may assume that $\left\{x_{1}, \ldots, x_{m}\right\}$ is Nielsen reduced. For this system we then have an equation

$$
\prod_{k=1}^{q} x_{\nu_{k}}^{\epsilon_{k}}=a_{1} \cdots a_{n}
$$

where $\epsilon_{k}= \pm 1, \epsilon_{k}=\epsilon_{k+1}$ if $\nu_{k}=\nu_{k+1}$.

Among the equations as in (1) there is one for which $q$ is minimal and let us assume that this is the case in Equation (1). Further we may also assume that each $x_{i} \neq 1$ and that each $x_{i}$ occurs in (1). If some $x_{i}$ occurs only once in (1) as either $x_{i}$ or $x_{i}^{-1}$ then case (1) of the theorem holds. That is $\left\{x_{1}, \ldots, x_{m}\right\}$ can be carried by a Nielsen transformation to a system 
$\left\{y_{1}, \ldots, y_{m}\right\}$ with $y_{1}=a_{1} \ldots a_{n}$. If this is not the case we will show that there is no $\lambda \in\{1, \ldots, m\}$ such that always

$$
L\left(x_{\nu}^{\epsilon} x_{\lambda} x_{\mu}^{\eta}\right)>L\left(x_{\nu}\right)-L\left(x_{\lambda}\right)+L\left(x_{\mu}\right)
$$

for $\nu, \mu \in\{1, \ldots, m\}, \epsilon, \eta= \pm 1$ and $\nu \neq \lambda \neq \mu$ or $\nu=\lambda \neq \mu, \epsilon=1$ or $\nu \neq \lambda=\mu, \eta=1$ or $\nu=\lambda=\mu, \epsilon=\eta=1$. It follows then that for $\lambda \in\{1, \ldots, m\}$ there is always some $\nu, \mu \in\{1, \ldots, m\}$ such that

$$
L\left(x_{\nu}^{\epsilon} x_{\lambda} x_{\mu}^{\eta}\right) \leq L\left(x_{\nu}\right)-L\left(x_{\lambda}\right)+L\left(x_{\mu}\right)
$$

for $\epsilon, \eta= \pm 1$ and $\nu \neq \lambda \neq \mu$ or $\nu=\lambda \neq \mu, \epsilon=1$ or $\nu \neq \lambda=\mu, \eta=1$ or $\nu=\lambda=\mu, \epsilon=\eta=1$. This means that each $x_{\lambda}$ is conjugate to some element of some $H_{s}$ and hence necessarily either we return to case (1) or case (2) holds proving the theorem.

We may assume that each $x_{i}$ either occurs twice in Equation (1) with the same exponent $\epsilon= \pm 1$ or occurs in (1) exactly once with exponent +1 and once with exponent -1 . In either case we always have

$$
L\left(x_{\nu_{k}}^{\epsilon_{k}} \cdots x_{\nu_{h}}^{\epsilon_{h}}\right) \geq L\left(x_{\nu_{l}}^{\epsilon_{l}}\right)
$$

for $1 \leq k \leq l \leq h \leq q$ and

$$
L\left(x_{\nu_{k}}^{\epsilon_{k}} x_{\nu_{k+1}}^{\epsilon_{k+1}} x_{\nu_{k+2}}^{\epsilon_{k+2}}\right) \geq L\left(x_{\nu_{k}}\right)-L\left(x_{\nu_{k+1}}\right)+L\left(x_{\nu_{k+2}}\right)
$$

for $1 \leq k \leq q-2$.

Assume that there is a $\lambda \in\{1, \ldots, m\}$ such that always

$$
L\left(x_{\nu}^{\epsilon} x_{\lambda} x_{\mu}^{\eta}\right)>L\left(x_{\nu}\right)-L\left(x_{\lambda}\right)+L\left(x_{\mu}\right)
$$

for $\nu, \mu \in\{1, \ldots, m\}, \epsilon, \eta= \pm 1$ and $\nu \neq \lambda \neq \mu$ or $\nu=\lambda \neq \mu, \epsilon=1$ or $\nu \neq \lambda=\mu, \eta=1$ or $\nu=\lambda=\mu, \epsilon=\eta=1$. Suppose in particular that $\lambda=\nu_{k}$. We write $u_{i}=x_{\nu_{i}}^{\epsilon_{i}}, 1 \leq i \leq q$. Let

$$
u_{i}=l_{i_{1}} \cdots l_{i_{m_{i}}} k_{i} r_{i_{m_{i}}} \cdots r_{i_{1}}
$$

be the symmetric normal form of $u_{i}$ (see $[\mathbf{Z 3}]$ and $[\mathbf{F}-\mathbf{R}-\mathbf{S}]$ ). We call

$$
l_{i_{1}}, \ldots, l_{i_{m_{i}}}, k_{i}, r_{i_{m_{i}}}, \ldots, r_{i_{1}}
$$

the places of $u_{i}$. For brevity we write $v_{i}$ for a place of $u_{i}$. In the following we write

$$
z \equiv z_{1} \cdots z_{p}
$$

to stand for the equality of the elements $=$ together with the fact that

$$
L(z)=L\left(z_{1}\right)+\cdots+L\left(z_{p}\right) .
$$

Given the $x_{\lambda}$ above and its places, there is an $a_{t}, 1 \leq t \leq n$, such that one of the following holds:

(a) $u_{k} \equiv p_{k} v_{k} q_{k}$ and $a_{t}=v_{k} b_{t}$ where $v_{k} \in H_{t} \backslash\{1\}$ is a place of $u_{k}$ and $b_{t}=1$ or $1 \neq b_{t} \in H_{t}$ and $u_{k+1} \cdots u_{k+l} \equiv q_{k}^{-1} b_{t} q_{k}$ for some $l$ with $1 \leq l \leq q-k$; 
(b) $u_{k} \equiv p_{k} v_{k} q_{k}$ and $a_{t}=b_{t} v_{k}$ where $v_{k} \in H_{t} \backslash\{1\}$ is a place of $u_{k}$ and $b_{t}=1$ or $1 \neq b_{t} \in H_{t}$ and $u_{k-l} \cdots u_{k-1} \equiv p_{k} b_{t} p_{k}^{-1}$ for some $l$ with $1 \leq l \leq k-1$

(c) $u_{k} \equiv p_{k} v_{k} q_{k}, u_{k+l+1} \equiv q_{k}^{-1} v_{k+l+1} q_{k+l+1}$ and $a_{t}=v_{k} b_{t} v_{k+l+1}$ where $0 \leq l \leq q-k-1, v_{k} \in H_{t} \backslash\{1\}$ is a place of $u_{k}, v_{k+l+1} \in H_{t} \backslash\{1\}$ is a place of $u_{k+l+1}$ and $b_{t}=1$ or $1 \neq b_{t} \in H_{t}$ and $u_{k+1} \cdots u_{k+l} \equiv q_{k}^{-1} b_{t} q_{k}$ for some $l$ with $1 \leq l \leq q-k-1$;

(d) $u_{k} \equiv p_{k} v_{k} q_{k}, u_{k-l-1} \equiv p_{k-l-1} v_{k-l-1} p_{k}^{-1}$ and $a_{t}=v_{k-l-1} b_{t} v_{k}$ where $0 \leq l \leq k-2, v_{k} \in H_{t} \backslash\{1\}$ is a place of $u_{k}, v_{k-l-1} \in H_{t} \backslash\{1\}$ is a place of $u_{k-l-1}$ and $b_{t}=1$ or $1 \neq b_{t} \in H_{t}$ and $u_{k-l} \cdots u_{k-1} \equiv p_{k} b_{t} p_{k}^{-1}$ for some $l$ with $1 \leq l \leq k-2$.

Note that if $u_{k+1} \ldots u_{k+l} \equiv q_{k}^{-1} b_{t} q_{k}$ or $u_{k-l} \ldots u_{k-1} \equiv p_{k} b_{t} p_{k}^{-1}, b_{t} \neq 1$, respectively then each $u_{i}$ occurring in this product is conjugate to an element of $H_{t}$.

To see all this assume that there is an $a_{t}$ with $1 \leq t \leq n$ for whose formation some $u_{i}, 1 \leq i<k$, and some $u_{j}, k<j \leq q$, contribute. Then $u_{k-1}$ cancels the whole leading half of $u_{k}$ and $u_{k+1}$ cancels the whole rear half of $u_{k}$ and the kernel of $u_{k}$ has a share in the formation of $a_{t}$. But then $L\left(u_{k-1} u_{k} u_{k+1}\right) \leq L\left(u_{k-1}\right)-L\left(u_{k}\right)+L\left(u_{k+1}\right)$ giving a contradiction.

Now suppose we have the blockwise description of $a_{t}$ above and we assume as before that $x_{\lambda}=x_{\nu_{k}}$ occurs twice in Equation (1). Then $\nu_{k}=\nu_{h}$ for some $h, 1 \leq h \leq q$, with $k \neq h$. Without loss of generality let $k<h$ and recall that all the $a_{t}, 1 \leq t \leq n$, are all different from different factors. If $\epsilon_{h}=\epsilon_{k}$ then the leading half of $x_{\lambda}=x_{\nu_{h}}=x_{\nu_{k}}$ is inverse to the rear half of $x_{\lambda}=x_{\nu_{k}}$, that is $x_{\nu_{k}}$ is conjugate to an element of some factor $H_{s}$ with $1 \leq s \leq n$. But then

$$
L\left(x_{\nu_{k}}^{3}\right) \leq L\left(x_{\nu_{k}}\right)=L\left(x_{\nu_{k}}\right)-L\left(x_{\nu_{k}}\right)+L\left(x_{\nu_{k}}\right)
$$

which gives a contradiction.

Now let $\epsilon_{h}=-\epsilon_{k}$. Then we have the following situation:

$u_{k} \equiv p_{k} v_{k} q_{k}, u_{h}=u_{k+l+1}=u_{k}^{-1} \equiv q_{k}^{-1} v_{k}^{-1} p_{k}^{-1}, u_{k+1} \cdots u_{k+l} \equiv q_{k}^{-1} b_{t} q_{k}$ with $v_{k}, b_{t} \in H_{t} \backslash\{1\}$ for some $t, 1 \leq t \leq n$ and some $l, 1 \leq l \leq q-k-1$. We may choose $v_{k}$ in such a way that $\left|L\left(p_{k}\right)-L\left(q_{k}\right)\right| \leq 1$. Assume that $p_{k} \neq 1$. Since the $a_{t}$ are all different from different factors, $p_{k}$ or $p_{k}^{-1}$ must be cancelled completely in Equation (1) by a $u_{i}$ with $i<k$ or $i>=h$ respectively, which is not conjugate to an element of some factor. For such an element $u_{i}$ we always have (see $[\mathbf{F}-\mathbf{R}-\mathbf{S}]$ )

$$
L\left(u_{l}^{\epsilon} u_{i} u_{j}^{\eta}\right)>L\left(u_{l}\right)-L\left(u_{i}\right)+L\left(u_{j}\right)
$$

for $l, j \in\{1, \ldots, q\}, \epsilon, \eta= \pm 1$ and $l \neq i \neq j$ or $l=i \neq j, \epsilon=1$ or $l \neq i=j, \eta=1$ or $l=i=j, \epsilon=\eta=1$. We have $i<k$ or $i>h$ so an inductive argument gives a contradiction because of the blockwise description of the $a_{t}$. 
Therefore we have $p_{k}=1$. This gives $q_{k} \neq 1, L\left(q_{k}\right)=1$ since $u_{k}$ is not conjugate to an element of some factor. But then $u_{k+1}=q_{k}^{-1} d_{t} q_{k}$ for some $d_{t} \in H_{t} \backslash\{1\}, u:=u_{k} u_{k+1} u_{k}^{-1}=v_{k} d_{t} v_{k}^{-1}$ and $L(u)=1<L\left(u_{k+1}\right)=3$ which contradicts the fact that $\left\{x_{1}, \ldots, x_{m}\right\}$ is Nielsen reduced. Hence $x_{\lambda}=x_{\nu_{k}}$ occurs only once in Equation (1) contradicting the assumption that each $x_{i}$ occurs twice in Equation (1). Therefore there is no $\lambda$ such that always

$$
L\left(x_{\nu}^{\epsilon} x_{\lambda} x_{\mu}^{\eta}\right)>L\left(x_{\nu}\right)-L\left(x_{\lambda}\right)+L\left(x_{\mu}\right)
$$

for $\nu, \mu \in\{1, \ldots, m\}, \epsilon, \eta= \pm 1$ and $\nu \neq \lambda \neq \mu$ or $\nu=\lambda \neq \mu, \epsilon=1$ or $\nu \neq \lambda=\mu, \eta=1$ or $\nu=\lambda=\mu, \epsilon=\eta=1$. As described before this statement completes the theorem.

We note the following. Suppose $\left\{x_{1}, \ldots, x_{m}\right\} \subset G=H_{1} \star \cdots \star H_{n}$, $m \geq 1, n \geq 2$, is a Nielsen reduced system as above with $x_{i} \neq 1$ for all $i$. Let $\lambda \in\{1, \ldots, m\}$ be such that always

$$
L\left(x_{\nu}^{\epsilon} x_{\lambda} x_{\mu}^{\eta}\right)>L\left(x_{\nu}\right)-L\left(x_{\lambda}\right)+L\left(x_{\mu}\right)
$$

for $\nu, \mu \in\{1, \ldots, m\}, \epsilon, \eta= \pm 1$ and $\nu \neq \lambda \neq \mu$ or $\nu=\lambda \neq \mu, \epsilon=1$ or $\nu \neq \lambda=\mu, \eta=1$ or $\nu=\lambda=\mu, \epsilon=\eta=1$. Let $w=a_{i_{1}} \cdots a_{i_{r}} \in G, r \geq 1$, be given in normal form and let $w \in H=\left\langle x_{1}, \ldots, x_{m}\right\rangle$. Let

$$
w=\prod_{k=1}^{q} x_{\nu_{k}}^{\epsilon_{k}}
$$

$\epsilon_{k}= \pm 1, \epsilon_{k}=\epsilon_{k+1}$ if $\nu_{k}=\nu_{k+1}$ with $q$ minimal. Assume that $x_{\lambda}$ occurs in this equation, for instance suppose $x_{\lambda}^{\epsilon_{k}}=x_{\nu_{k}}^{\epsilon_{k}}=: u_{k}$. Then there is an $a_{i_{j}}$ related to $u_{k}=x_{\lambda}^{\epsilon}$ which is described via the block relation to a place $v_{k}$ of $u_{k}$ as in the proof of the theorem. Such a $v_{k}$ is called a semistable letter of $u_{k}$. The advantage of a semistable letter is that it can be influenced in such an equation as above, only from one side.

Using the theorem we obtain the following result on APE's in free products of free groups.

Theorem 3. Let $F$ be a finitely generated free group with basis $B$. Let $B_{1}, \ldots, B_{n}, n \geq 2$, be pairwise disjoint, non-empty subsets of $B$ and let $F_{j}$ be the subgroup of $F$ generated by $B_{j}, 1 \leq j \leq n$. Let $a_{j} \in F_{j}$ with $a_{j} \neq 1$, $1 \leq j \leq n$ and let $a=a_{1} \ldots a_{n}$. Then:

(1) If each $a_{j}$ is an APE in $F_{j}$ then a is an APE in F.

(2) Let $\mathcal{U}$ a non-trivial variety defined by the set of laws $\mathcal{V}$.

(a) Let $a_{j} \in \mathcal{V}\left(F_{j}\right)$. If each $a_{j}$ is $\mathcal{U}$-generic in $F_{j}$ then $a \in \mathcal{V}(F)$ and $a$ is $\mathcal{U}$-generic in $F$.

(b) Let $a \in \mathcal{V}(F)$. If a is $\mathcal{U}$-generic in $F$ then each $a_{j} \in \mathcal{V}\left(F_{j}\right)$ and each $a_{j}$ is $\mathcal{U}$-generic in $F_{j}$.

(3) (a) Let $a_{j} \in F_{j}^{q} F_{j}^{\prime}, q=0$ or $q=2$, for each $j, 1 \leq j \leq n$. If each $a_{j}$ is a test word in $F_{j}$ then $a$ is a test word in $F$. 
(b) Let $a \in F^{q} F^{\prime}, q=0$ or $q=2$. If a is a test word in $F$ then each $a_{j}$ is a test word in $F_{j}$.

Proof. (1) Let $a_{j} \in F_{j}$ with $a_{j} \neq 1,1 \leq j \leq n$, and let $a=a_{1} \cdots a_{n}$. Then $a$ cannot be primitive in $F$ because in that case at least one $a_{j}$ has to be primitive in $F_{j}$ contradicting that each $a_{j}$ is an APE. Let $K$ be a proper subgroup of $F$ with $a \in K$. From Theorem 2, $a$ is primitive in $K$ or without loss of generality, we may assume that $K$ has a finite basis $X$ which is the disjoint union of $n$ subsets $X_{j}$ of $F_{j}$ such that $a_{j} \in K_{j} \subset F_{j}$ for each $j, 1 \leq j \leq n$, where $K_{j}$ is the subgroup generated by $X_{j}$. We consider this latter situation. If $K_{j}=F_{j}$ for each $j$ then $K=\left\langle K_{1}, \ldots, K_{n}\right\rangle$ $=\left\langle F_{1}, \ldots, F_{n}\right\rangle=F$ contradicting the fact that $K \neq F$. Hence $K_{j}$ is a proper subgroup of $F_{j}$ for at least one $j$. Suppose $K_{1} \subset F_{1}, K_{1} \neq F_{1}$. Then $a_{1}$ is primitive in $K_{1}$ since $a_{1}$ is an APE in $F_{1}$ and hence $a=a_{1} \cdots a_{n}$ is primitive in $K$. This completes part (1).

(2)(a) Let each $a_{j}$ be $\mathcal{U}$-generic in $F_{j}$. Since each $a_{j} \in \mathcal{V}\left(F_{j}\right)$ we have $a \in \mathcal{V}(F)$. Let $\phi: H \rightarrow F$ be a homomorphism with $\phi(u)=a$ for some $u \in \mathcal{V}(H)$. Without loss of generality assume $H$ to be finitely generated. $a$ cannot be primitive in $K=\phi(H)$ because $\mathcal{U}$ is non-trivial. Let $A$ be a finite generating system for $H$. Then $X=\phi(A)$ is a finite generating system for $K$. We apply Theorem 2 and the fact that a Nielsen transformation from $X$ to a system $Y$ defines an epimorphism from $K$ onto $K$. Hence without loss of generality we assume that $X$ is the disjoint union of $n$ subsets $X_{j}$ of $F_{j}$ such that $a_{j} \in K_{j} \subset F_{j}$ for each $j$ with $1 \leq j \leq n$ where $K_{j}$ is the subgroup generated by $X_{j}$. Let $H_{j}=\phi^{-1}\left(K_{j}\right)$ for each $j$. Then $\phi_{j}=\phi_{\mid H_{j}}$ defines a homomorphism $\phi_{j}: H_{j} \rightarrow F_{j}$ with $a_{j}=\phi_{j}\left(u_{j}\right), 1 \leq j \leq n$, for some $u_{j} \in \mathcal{V}\left(H_{j}\right)$. Since each $a_{j}$ is $\mathcal{U}$-generic, $\phi_{j}$ is an epimorphism for each $j$. Therefore $\phi$ is an epimorphism completing part (2)(a).

(2)(b) Certainly each $a_{j} \in \mathcal{V}\left(F_{j}\right)$ if $a \in \mathcal{V}(F)$. For each $j$ let $\phi_{j}: H_{j} \rightarrow F_{j}$ be a homomorphsim from some group $H_{j}$ such that $\phi\left(u_{j}\right)=a_{j}$ for some $u_{j} \in \mathcal{V}\left(H_{j}\right)$. Let $H=H_{1} \star \cdots \star H_{n}$ and let $\phi: H \rightarrow F$ be the induced homomorphism with $\phi_{\mid H_{j}}=\phi_{j}$. Then $\phi\left(u_{1} \ldots u_{n}\right)=\phi\left(a_{1} \cdots a_{n}\right)=\phi(a)$ and $u_{1} \ldots u_{n} \in \mathcal{V}(H)$. Since $a$ is $\mathcal{U}$-generic, $\phi$ is an epimorphism. Hence each $\phi_{j}$ is an epimorphism and therefore each $a_{j}$ is $\mathcal{U}$-generic. This completes part (2)(b).

(3)(a) For a group $G$ we let $G^{q} G^{\prime}, q=0$ or $q \geq 2$, denote the subgroup of $G$ generated by the $q$-th powers and the commutators in $G$.

Let each $a_{j}$ be a testword in $F_{j}$ and suppose each $a_{j} \in F_{j}^{q} F_{j}^{\prime}, q=0$ or $q \geq 2$. Then $a \in F^{q} F^{\prime}, q=0$ or $q \geq 2$. Let $\phi: F \rightarrow F$ be an endomorphism with $\phi(a)=a$. Let $K=\phi(F)$. Since $a \in F^{q} F^{\prime}$ we also have $a \in K^{q} K^{\prime}, q=0$ or $q \geq 2$. Hence $a$ is not primitive in $K$ and we must show that $K=F$.

Let $X=\phi(B)$. We show first that $X$ is a basis for $K$. From Theorem 2, $X$ can be carried by a Nielsen transformation, relative to $a=a_{1} \cdots a_{n}$ into 
a free basis $Y$ of $K$ which contains a subset $Z$ which is the disjoint union of $n$ subsets $Z_{j}$ of $F_{j}$ such that $a_{j} \in K_{j} \subset F_{j}$ for each $j, 1 \leq j \leq n$, where $K_{j}$ is the subgroup generated by $Z_{j}$. Since each $a_{j}$ is a testword in $F_{j}$ we must have $\left|Z_{j}\right|=\left|B_{j}\right|$ for each $j$ and hence $|Z|=|B|$. This gives

$$
|Z| \leq|Y| \leq|X| \leq|B|=|Z|
$$

and hence $Y=Z$ and $X$ is a basis of $K$. Now the Nielsen transformation from $X$ to the above system $Y$ defines an automorphsim $\alpha$ of $K$. Hence we may already assume that $X=Y=Z$ because of the free product decomposition $F=F_{1} \star \cdots \star F_{n}$ and the description of $a$ as $a=a_{1} \cdots a_{n}$. Starting, with a permutation of $B$, if necessary, we obtain this way an endomorphism $\psi: F \rightarrow F$ such that $\psi_{j}=\psi_{\mid F_{j}}$ defines an endomorphism $\psi_{j}: F_{j} \rightarrow F_{j}$ with $\psi_{j}\left(a_{j}\right)=a_{j}$ for $1 \leq j \leq n$. Since each $a_{j}$ is a testword in $F_{j}$ we have that each $\psi_{j}$ is an automorphism of $F_{j}$. Hence by combination, $\psi$ is an automorphism of $F$. Therefore by construction, $\phi$ is also an automorphism of $F$ and it follows that $a$ is a testword in $F$.

(3)(b) Since $a \in F^{q} F^{\prime}, q=0$ or $q \geq 2$, we have $a_{j} \in F_{j}^{q} F_{j}^{\prime}, q=0$ or $q \geq 2$. For each $j$ let $\phi_{j}: F_{j} \rightarrow F_{j}$ be an endomorphsim with $\phi_{j}\left(a_{j}\right)=a_{j}$. Then $\phi: F \rightarrow F$ with $\phi_{\mid F_{j}}=\phi_{j}$ defines an endomorphism $\phi: F \rightarrow F$ with $\phi(a)=\phi\left(a_{1} \cdots a_{n}\right)=a_{1} \cdots a_{n}=a$. Since $a$ is a testword in $F, \phi$ is an automorphism of $F$. Hence each $\phi_{j}$ is an automorphism of $F_{j}$ and therefore each $a_{j}$ is a testword in $F_{j}$. This completes the theorem.

Corollary 2. Let $F=\left\langle x_{1}, y_{1}, \ldots, x_{g}, y_{g} ;\right\rangle, g \geq 1$. Let $a_{j}=a_{j}\left(x_{j}, y_{j}\right) \neq 1$ for $j=1, \ldots, g$ and let both $x_{j}$ and $y_{j}$ occur in the freely reduced expression of $a_{j}$. Let $\left|a_{j}\right|_{x_{j}}$ be the total exponent sum of $x_{j}$ in $a_{j}$ and let $\left|a_{j}\right|_{y_{j}}$ be the total exponent of $y_{j}$ in $a_{j}$. Let $F_{j}$ be the subgroup generated by $x_{j}$ and $y_{j}$.

(1) Let each $a_{j}$ be not a proper power in $F_{j}$. Then a is a testword in $F$ if and only if $\operatorname{gcd}\left(\left|a_{j}\right|_{x_{j}},\left|a_{j}\right|_{y_{j}}\right) \neq 1$ for each $j$.

(2) Let $a$ be an element of the commutator subgroup of $F$ and suppose a is a product $a=a_{1} a_{2} \cdots a_{g}$ where each $a_{j}$ is a non-trivial element of the commutator subgroup in $F_{j}$. Then a is a testword.

Proof. This follows directly from Theorem 3 and Example 4 in Turner's paper $[\mathbf{T}]$.

\section{A Class of Generic Elements.}

In this section we give a class of examples of generic elements.

Theorem 4. Let $F$ be a free group on $a, b$ and let $X=\left\langle x_{1}, \ldots, x_{k}\right\rangle, k \geq 1$ be a finitely generated subgroup of $F$. Suppose that $X$ contains the element $\left[a^{n}, b^{m}\right]$ for positive integers $n, m$. Then $\left\{x_{1}, \ldots, x_{k}\right\}$ can be carried by a Nielsen transformation into a free basis $\left\{y_{1}, \ldots, y_{p}\right\}, 1 \leq p \leq k$, for $X$ for which one of the following cases occurs. 
(1) $y_{1}=\left[a^{n}, b^{m}\right]$ is a primitive element of $X$;

(2) $y_{1}=a^{\alpha}, 1 \leq \alpha \leq n, \alpha \mid n$ and $y_{2}=b^{\beta}, 1 \leq \beta \leq m, \beta \mid m$;

(3) $y_{1}=a^{\alpha}, 1 \leq \alpha \leq n, \alpha \mid n$ and $y_{2}=b^{m} a^{\beta} b^{-m}, 1 \leq \beta \leq n, \beta \mid n ;$

(4) $y_{1}=b^{\alpha}, 1 \leq \alpha<m, \alpha \mid m$ and $y_{2}=a^{n} b^{\beta} a^{-n}, 1 \leq \beta \leq m, \beta \mid m ;$

(5) $y_{1}=a^{\alpha}, 1 \leq \alpha \leq n, \alpha \mid n$ and $y_{2}=b^{m} a^{\beta}, 1 \leq \beta<\alpha$

(6) $y_{1}=b^{\alpha}, 1 \leq \alpha \leq m, \alpha \mid m$ and $y_{2}=a^{n} b^{\beta}, 1 \leq \beta<\alpha$

(7) $y_{1}=a^{n} b^{m}, y_{2}=a^{\alpha}, 1 \leq \alpha \leq 2 n, \alpha \mid 2 n$ and $y_{3}=b^{\beta}, 1 \leq \beta \leq 2 m, \beta \mid 2 m$.

Proof. The proof follows the general outline of the proof of Theorem 2 . Regard $F$ as the free product $F=\langle a\rangle \star\langle b\rangle$ together with the length $L$ and order with respect to this factorization. We may assume $\left\{x_{1}, \ldots, x_{k}\right\}$ is Nielsen reduced with $x_{i} \neq 1$ for all $i$. Further we may assume from the start that there is no Nielsen transformation from $\left\{x_{1}, \ldots, x_{k}\right\}$ to a system $\left\{y_{1}, \ldots, y_{k}\right\}$ with $\left[a^{n}, b^{m}\right] \in\left\langle y_{1}, \ldots, y_{k-1}\right\rangle$, that is $k$ is minimal with respect to this property.

As in the proof of Theorem 2, for this system we then have an equation

$$
\prod_{k=1}^{q} x_{\nu_{k}}^{\epsilon_{k}}=\left[a^{n}, b^{m}\right]
$$

where $\epsilon_{k}= \pm 1, \epsilon_{k}=\epsilon_{k+1}$ if $\nu_{k}=\nu_{k+1}$.

Among the equations as in (2) there is one for which $q$ is minimal and let us assume that this is the case in Equation (2). Further we may also assume that each $x_{i} \neq 1$ and that each $x_{i}$ occurs in (2). If some $x_{i}$ occurs only once in (2) as either $x_{i}$ or $x_{i}^{-1}$ then case (1) of the theorem holds. Therefore for the rest of the proof we assume that case (1) does not hold.

Hence each $x_{i}$ either occurs twice in Equation (2) with the same exponent $\epsilon= \pm 1$ or occurs in (2) exactly once with exponent +1 and once with exponent -1 . In either case we always have

$$
L\left(x_{\nu_{k}}^{\epsilon_{k}} \cdots x_{\nu_{h}}^{\epsilon_{h}}\right) \geq L\left(x_{\nu_{l}}^{\epsilon_{l}}\right)
$$

for $1 \leq k \leq l \leq h \leq q$ and

$$
L\left(x_{\nu_{k}}^{\epsilon_{k}} x_{\nu_{k+1}}^{\epsilon_{k+1}} x_{\nu_{k+2}}^{\epsilon_{k+2}}\right) \geq L\left(x_{\nu_{k}}\right)-L\left(x_{\nu_{k+1}}\right)+L\left(x_{\nu_{k+2}}\right)
$$

for $1 \leq k \leq q-2$. Especially, we have $L\left(x_{i}\right) \leq 4$ for all $i$. Since we have only two cyclic factors and since $\left\{x_{1}, \ldots, x_{k}\right\}$ is Nielsen reduced, for each $x_{i}$, which is not conjugate to a power of $a$ or $b$, we have at least two places which are semistable letters for this $x_{i}$. This excludes the possibility $L\left(x_{i}\right)=4$. The blockwise decription as in the proof of Theorem 2, together 
with $L\left(\left[a^{n}, b^{m}\right]\right)=4$ gives that there is at most one $x_{i}$ which is not conjugate to a power of $a$ or $b$, and which occurs in (2) exactly twice with the same exponent or exactly once with exponent +1 and once with exponent -1 . Also, if there is such an $x_{i}$ it must have length two.

Now suppose first there is an $x_{i}$ which is not conjugate to a power of $a$ or $b$, and which occurs in (2) exactly once with exponent +1 and once with exponent -1 . Suppose that $x_{i}=a^{\alpha_{1}} b^{\beta_{1}}$. Then, since $L\left(\left[a^{n}, b^{m}\right]\right)=4$ the other $x_{j}$ are powers of $a$ or $b$. Recall that if we have, for instance, powers $a^{\alpha_{1}}, \ldots, a^{\alpha_{p}}, p \geq 2$, then there is a Nielsen transformation from $\left\{a^{\alpha_{1}}, \ldots, a^{\alpha_{p}}\right\}$ to $\left\{a^{\gamma}, 1, \ldots, 1\right\}$ with $\gamma=\operatorname{gcd}\left(\alpha_{1}, \ldots, \alpha_{p}\right)$. Hence by the minimality of $k$, it follows that no two of the $x_{i}$ are powers of $a$ and no two of the $x_{i}$ are powers of $b$. A typical possible situation to consider is now, after some renumbering,

$$
\begin{aligned}
x_{1}^{\alpha_{0}} x_{2} x_{3}^{\beta_{0}} x_{2}^{-1} x_{1}^{\alpha_{2}} x_{3}^{\beta_{2}} & =a^{\gamma_{1} \alpha_{0}} a^{\alpha_{1}} b^{\beta_{1}} b^{\gamma_{2} \beta_{0}} b^{-\beta_{1}} a^{-\alpha_{1}} a^{\gamma_{1} \alpha_{2}} b^{\gamma_{2} \beta_{2}} \\
& =a^{n} b^{m} a^{-n} b^{-m}
\end{aligned}
$$

with $\alpha_{0} \neq 0 \neq \alpha_{2}$. Then, necessarily $\gamma_{1} \alpha_{0}+\alpha_{1}=n, \gamma_{2} \beta_{0}=m, \gamma_{1} \alpha_{2}-\alpha_{1}=$ $-n$ and $\gamma_{2} \beta_{2}=-m$. In particular we have $\alpha_{0}=-\alpha_{2}$ and via a Nielsen transformation we may replace $x_{2}$ by $x_{1}^{\alpha_{0}} x_{2}=a^{\gamma_{1} \alpha_{0}} a^{\alpha_{1}} b^{\beta_{1}}=a^{n} b^{\beta}$. But this contradicts the minimality of $k$. Therefore the above possible situation reduces to, again after some renumbering, to the situation

$$
x_{1}=a^{n} b^{\beta_{1}}, x_{2}=b^{\gamma_{2}} \text { and } x_{1} x_{2}^{\beta_{0}} x_{1}^{-1} x_{2}^{-\beta_{0}}=\left[a^{n}, b^{m}\right]
$$

because $\beta_{0}=-\beta_{2}$. We may reduce $\beta_{1}$ to a $\beta$ with $1 \leq \beta<\gamma_{2}$ via a Nielsen transformation and obtain case (6) in the theorem.

An analogous case by case consideration gives that we obtain either case (5) or case (6) if there is an $x_{i}$ which is not conjugate to a power of $a$ or $b$ and which occurs in (2) exactly once with exponent +1 and once with exponent -1 . If each $x_{i}$ is conjugate to a power of $a$ or $b$ we obtain cases (2), (3) or (4) since again no two of the $x_{i}$ are powers of $a$ and no two of the $x_{i}$ are powers of $b$.

Finally suppose that one $x_{i}$ is not conjugate to a power of $a$ or $b$ and occurs twice with the same exponent. Without loss of generality assume this exponent to be +1 . Because of $L\left(\left[a^{n}, b^{m}\right]\right)=4$ and the blockwise description as given in the proof of Theorem 2, this $x_{i}$ occurs exactly twice and has length 2 and recall that no other $x_{l}$ occurs which is not a power of $a$ or $b$. Let $x_{i}=a^{\gamma} b^{\delta}, \gamma \neq 0, \delta \neq 0$. Then after renumbering we get an equation

$$
x_{1}^{\alpha_{1}} x_{2} x_{3}^{\beta_{1}} x_{1}^{\alpha_{2}} x_{2} x_{3}^{\beta_{2}}=\left[a^{n}, b^{m}\right]
$$

with $x_{1}=a^{\gamma_{1}}, x_{3}=b^{\delta_{1}}, x_{2}=a^{\gamma} b^{\delta}$. We may assume that $\gamma_{1} \geq 1$ and $\delta_{1} \geq 1$. We have necessarily

$$
\gamma_{1} \alpha_{1}+\gamma=n, \delta_{1} \beta_{1}+\delta=m, \gamma_{1} \alpha_{2}+\gamma=-n \text { and } \delta_{1} \beta_{2}+\delta=-m .
$$


Then $\gamma_{1} \mid 2 n$ and $\delta_{1} \mid 2 m$, and $1 \leq \gamma_{1} \leq 2 n, 1 \leq \delta_{1} \leq 2 m$. If we replace $x_{2}$ by $y_{2}=x_{1}^{\alpha_{1}} x_{2} x_{3}^{\beta_{1}}$ this defines a Nielsen transformation and we obtain an equation

$$
y_{2} x_{1}^{\alpha_{2}-\alpha_{1}} y_{2} x_{3}^{\beta_{2}-\beta_{1}}=\left[a^{n}, b^{m}\right]
$$

with $y_{2}=a^{n} b^{m}, x_{1}=a^{\gamma_{1}}, 1 \leq \gamma_{1} \leq 2 n, \gamma_{1} \mid 2 n$ and $x_{3}=b^{\delta_{1}}, 1 \leq \delta_{1} \leq$ $2 m, \delta_{1} \mid 2 m$. Since our system is Nielsen reduced we have that $\gamma_{1}$ does not divide $n$ and $\delta_{1}$ does not divide $m$. This gives case (7).

The case $x_{i}=b^{\delta} a^{\gamma}, \delta \neq 0, \gamma \neq 0$, cannot occur since the exponent is +1 and $\left[a^{n}, b^{m}\right]$ starts with a power of $a$ and ends with a power of $b$.

Using the theorem we first obtain the following corollaries. The first is due to Comerford and Edmonds $[\mathbf{C}-\mathbf{E}]$ and the second due to Turner $[\mathbf{T}]$.

Corollary 3. Let $F$ be the free group on $x, y$ and let $\left[x_{1}, x_{2}\right]=\left[x^{n}, y^{m}\right]$, $n, m \geq 1$. Then $\left\{x_{1}, x_{2}\right\}$ is Nielsen equivalent to a pair $\left\{y_{1}, y_{2}\right\}$ with either $y_{1}=x^{n}$ and $y_{2}=y^{m} x^{\alpha}, 0 \leq \alpha<n$ or $y_{1}=y^{m}$ and $y_{2}=x^{n} y^{\beta}, 1 \leq \beta<m$.

Corollary 4. The element $\left[x^{n}, y^{m}\right]$ is a test word in the free group of rank two on $x, y$ for any $n, m \geq 1$.

Recall that $\mathcal{U}_{n}$ is the variety generated by the laws $\mathcal{V}_{n}=\left\{[x, y], z^{n}\right\}, n=$ 0 or $n \geq 2$. We let $\mathcal{L}_{n}$ be the variety generated by the laws $\mathcal{W}_{n}=$ $\left\{\left[x^{n}, y^{n}\right]\right\}, n \geq 1$. We then obtain the following class of generic elements.

Corollary 5. Let $F$ be a free group of rank 2 on $x, y$. Then $\left[x^{n}, y^{n}\right]$ is $\mathcal{L}_{n}$-generic in $F$ but for $n \geq 2$ it is not $\mathcal{U}_{n}$-generic in $F$.

Corollary 6. Let $F$ be a free group of rank 2 on $x, y$. Then the element $\left[x^{n}, y^{m}\right], n, m \geq 1$, is an APE if and only if $n=m=1$.

Recall that in general it is not true that being an APE implies being a test word. As mentioned earlier if $F=F(x, y)$ and $w=x^{2} y x^{-1} y^{-1}$ then $w$ is an APE but is not a test word. Since generic elements are test words this example shows further that APE does not imply generic in general. However using the same techniques as in Theorem 4 we can generalize the fact that the element $w$ above is an APE to obtain further examples of APE's and testwords.

Theorem 5. Let $F=\langle a, b ;\rangle$ and let $X=\left\langle x_{1}, \ldots, x_{k}\right\rangle \subset F, k \geq 1$. Suppose $a^{n} b a^{-1} b^{-1} \in X, n \geq 2$. Then there is a Nielsen transformation from $\left\{x_{1}, \ldots, x_{k}\right\}$ to a basis $\left\{y_{1}, \ldots, y_{p}\right\}, 1 \leq p \leq k$, of $X$ such that one of the following cases holds:

(1) $y_{1}=a^{n} b a^{-1} b^{-1}$ or

(2) $y_{1}=a, y_{2}=b$.

From this theorem and Theorem 1 we get the following corollary.

Corollary 7. Let $F=\langle a, b ;\rangle$. Then 
(1) $a^{n} b a^{-1} b^{-1}, n \geq 2$, is an $A P E$;

(2) $a^{n} b a^{-1} b^{-1}, n \geq 3$, is $\mathcal{U}_{n-1}$-generic;

(3) $a^{n} b a^{-1} b^{-1}, n \geq 3$, is a testword in $F$;

(4) $a^{2} b a^{-1} b^{-1}$ is not a testword in $F$.

Proof of Theorem 5. The proof follows the same outline as the proof of Theorem 4. Assume that $\left\{x_{1}, \ldots, x_{k}\right\}$ is Nielsen reduced and $k$ is minimal in the sense that $\left\{x_{1}, \ldots, x_{k}\right\}$ is not Nielsen equivalent to a system $\left\{y_{1}, \ldots, y_{k}\right\}$ with $a^{n} b a^{-1} b^{-1} \in\left\langle y_{1}, \ldots, y_{k-1}\right\rangle$. Assume further that each $x_{i}$ occurs at least twice in the freely reduced equation expressing $a^{n} b a^{-1} b^{-1}$ in terms of $x_{1}, \ldots, x_{k}$. Assume that case (1) does not hold and assume that there is one $x_{i}$ which is not conjugate to a power of $a$ or $b$. Suppose first that this $x_{i}$ occurs twice with the same exponent, without loss of generality say +1 . As in the proof of Theorem 4, it follows from $L\left(a^{n} b a^{-1} b^{-1}\right)=4$, the fact that the system is Nielsen reduced and the blockwise description that this $x_{i}$ occurs exactly twice, has length 2 , and no other $x_{l}$ occurs which is not a power of $a$ or $b$. Let $x_{i}=a^{\gamma} b^{\delta}, \alpha \neq 0 \neq \delta$. As in Theorem $4, x_{i}=b^{\delta} a^{\gamma}$ cannot occur. Then after renumbering we obtain an equation

$$
x_{1}^{\alpha_{1}} x_{2} x_{3}^{\beta_{1}} x_{1}^{\alpha_{2}} x_{2} x_{3}^{\beta_{2}}=a^{n} b a^{-1} b^{-1}
$$

with $x_{1}=a^{\gamma_{1}}, x_{3}=b^{\delta_{1}}, x_{2}=a^{\gamma} b^{\delta}$. We may assume that $1 \leq \delta_{1}, 1 \leq \gamma_{1}$, $1 \leq \gamma<\gamma_{1}$ and $1 \leq \delta<\delta_{1}$. Then necessarily $\delta+\beta_{1} \delta_{1}=1$ and $\delta+\beta_{2} \delta_{1}=-1$. Hence $\delta_{1}=1$ or 2 . Since the system is Nielsen reduced $\delta_{1} \neq 1$ and hence $\delta_{1}=2$. Then $\delta=1$ and hence $\beta_{1}=0$ and $\beta_{2}=-1$. Thus we have $x_{1}^{\alpha_{1}} x_{2} x_{1}^{\alpha_{2}} x_{2} x_{3}^{-1}=a^{n} b a^{-1} b^{-1}$ contradicting the assumption that case (1) does not hold. It follows therefore that this $x_{i}$ occurs exactly once with exponent +1 and once with exponent -1 . Then as in the proof of Theorem 4 we must consider equations of the form

$$
x_{1}^{\alpha_{1}} x_{2} x_{3}^{\beta_{1}} x_{2}^{-1} x_{1}^{\alpha_{2}} x_{3}^{\beta_{2}}=a^{n} b a^{-1} b^{-1}
$$

with $x_{1}=a^{\gamma_{1}}, x_{3}=b^{\delta_{1}}, x_{2}=a^{\gamma} b^{\delta}$ and $0 \neq \gamma, 0 \neq \delta$. Without loss of generality let $1 \leq \gamma_{1}, 1 \leq \delta_{1},\left(\gamma_{1}=0\right.$ or $\delta_{1}=0$ cannot occur since $\left.n \geq 2\right)$. Then necessarily $\delta_{1} \beta_{1}=1$ and hence $\delta_{1}=1$. But this contradicts the fact that the system is Nielsen reduced since we can replace $x_{2}$ by $x_{2} x_{3}^{-\delta}=$ $a^{\gamma} b^{\delta} b^{-\delta}=a^{\gamma}$. The other possibilities are analogous. If each $x_{i}$ is conjugate to a power of $a$ or $b$ then case (2) certainly holds if case (1) does not. This completes the proof of the theorem.

The corollary now follows easily. If $w=a^{n} b a^{-1} b^{-1}$ is in any proper subgroup of $F$ then condition (2) of the theorem cannot hold and hence condition (1) must hold, that is $w$ is primitive. Therefore $w$ is an APE. If $n \geq 3$ then $w \in \mathcal{V}_{n-1}(F)$ where $\mathcal{V}_{n}$ is the set of laws $\mathcal{V}_{n}=\left\{[x, y], z^{n}\right\}$. As 
before if $\mathcal{U}_{n}$ is the variety defined by this set of laws, then $\mathcal{U}_{n}$ is an nontrivial variety and it follows that $w$ is an APE and that $w$ is $\mathcal{U}_{n-1}$-generic and hence a testword. Finally part (4) comes from Turner $[\mathbf{T}]$.

\section{A Result on Varieties and Primitive Elements.}

The following result relates when the laws determined by a single element generate a trivial variety and being in a retract.

Theorem 6. Let $F$ be the free group on $x_{1}, \ldots, x_{n}$ with $n \geq 2$ and let $w$ be a freely reduced non-empty word in the generators of $F$ which does not define a proper power of $F$. Then if the law $w=1$ determines the trivial variety (consisting only of trivial groups) then $w$ is a primitive in a retract of $F$.

Proof. Notice first that if $\mathcal{B}$ is a non-trivial variety then since $\mathcal{B}$ is closed under subgroup formation it must contain non-trivial cyclic groups. Hence the intersection of $\mathcal{B}$ with the abelian variety $\mathcal{A}$ is not the trivial variety.

Now let $\mathcal{V}$ be the variety determined by the law $w=1$. Let $\mathcal{E}$ stand for the trivial variety. Then $\mathcal{V}=\mathcal{E}$ is equivalent to the following two conditions being simultaneously satsified:

(1) $w \notin[F, F]=F^{\prime}$ and

(2) If $e_{i}$ is the exponent sum in $w$ of $x_{i}, i=1, \ldots, n$, then $\operatorname{gcd}\left(e_{1}, \ldots, e_{n}\right)$ $=1$.

To see this suppose that (1) and (2) are satisfied by $w$. Since $w \equiv x_{1}^{e_{1}} \ldots$ $x_{n}^{e_{n}}(\bmod [F, F])(1)$ is equivalent to $\left(e_{1}, \ldots, e_{n}\right) \neq(0, \ldots, 0)$. Let $m_{1}, \ldots$, $m_{n}$ be integers such that $m_{1} e_{2}+\cdots+m_{n} e_{n}=1$. Let $G$ be an abelian group lying in $\mathcal{V}$. Then $G$ must satisfy the law $x_{1}^{e_{1}} \cdots x_{n}^{e_{n}}=1$. Let $x \in G$. Let $x_{i}=x^{m_{i}}$. Then from the law $x^{m_{1} e_{1}+\cdots+m_{n} e_{n}}=x=1$. Therefore $G$ is trivial. Hence $\mathcal{V}$ contains no non-trivial abelian groups and therefore it follows from the remark above that $\mathcal{V}$ is itself trivial.

Conversely suppose $\mathcal{V}$ is trivial. We show that conditions (1) and (2) must hold. Suppose (1) does not hold so that $w \in[F, F]$. Let $c_{1}, \ldots, c_{n}$ be arbitrary integers. Then the infinite cyclic group $A=\langle a ;\rangle$ lies in $\mathcal{V}$ since $w\left(a^{c_{1}}, \ldots, a^{c_{n}}\right) \in[A, A]=1$. This contradicts the triviality of $\mathcal{V}$ so therefore (1) must hold.

Now suppose $w \notin[F, F]$ but $(2)$ is violated. Suppose $\operatorname{gcd}\left(e_{1}, \ldots, e_{n}\right)=$ $d>1$. Then the finite cyclic group $B=\left\langle b ; b^{d}=1\right\rangle$ lies in $\mathcal{V}$ since for any integers $c_{1}, \ldots, c_{n}$,

$$
w\left(b^{c_{1}}, \ldots, b^{c_{n}}\right)=\left(b^{c_{1}}\right)^{e_{1}} \cdots\left(b^{c_{n}}\right)^{e_{n}}=b^{c_{1} e_{1}+\cdots+c_{n} e_{n}}=1
$$

and $d \mid c_{1} e_{1}+\cdots+c_{n} e_{n}$. Since $d>1, B$ is non-trivial contradiciting the triviality of $\mathcal{V}$ so therefore (2) must also hold.

Now suppose $w$ satisfies (1) and (2) and $m_{1}, \ldots, m_{n}$ are integers such that $m_{1} e_{1}+\cdots+m_{n} e_{n}=1$. Consider the map $F \rightarrow\langle w\rangle$ given by $x_{1} \rightarrow$ 
$w^{m_{1}}, \ldots, x_{n} \rightarrow w^{m_{n}}$. Suppose $w=x_{i_{1}}^{k_{1}} \cdots x_{i_{l}}^{k_{l}}$ where each $k_{j}$ is a non-zero integer with $i_{j} \neq i_{j+1}$ for $j=1, \ldots, l-1$. Then under the above map

$$
\begin{aligned}
w \rightarrow w^{m_{i_{1}} k_{1}+\cdots+m_{i_{l}} k_{l}} & =w^{m_{1} \sum_{i_{j}=1} k_{j}+\cdots+m_{n} \sum_{i_{j}=n} k_{j}} \\
& =w^{m_{1} e_{1}=\cdots+m_{n} e_{n}}=w .
\end{aligned}
$$

It follows that $F \rightarrow\langle w\rangle$ is a retraction and clearly $w$ is primitive in $\langle w\rangle$.

The above proof depended on the fact that if $\mathcal{A}$ is the abelian variety and $\mathcal{E}$ is the trivial variety then $\mathcal{B} \cap \mathcal{A}=\mathcal{E}$ implies that $\mathcal{B}=\mathcal{E}$. The next result completely characterizes the varieties such as $\mathcal{A}$ with this property. Recall that a variety $\mathcal{V}$ has exponent $n$ if it satisfies the law $X^{n}=1$. If $\mathcal{V}$ has no finite exponent it has infinite exponent.

Theorem 7. Let $\mathcal{V}$ be a variety. Then $\mathcal{V}$ has the property that $\mathcal{B} \cap \mathcal{V}=\mathcal{E}$ implies that $\mathcal{B}=\mathcal{E}$ for an arbitrary variety $\mathcal{B}$ if and only if $\mathcal{V}$ has infinite exponent.

Proof. Suppose $\mathcal{V}$ has infinite exponent. Therefore $\mathcal{V}$ contains infinite cyclic groups and since it is closed under the formation of quotients it contains cyclic groups of all possible finite orders. Since any non-trivial variety must contain cyclic groups of some order it follows that $\mathcal{V}$ will intersect nontrivially with any non-trivial variety.

Conversely suppose $\mathcal{V}$ has the stated property. If $\mathcal{V}$ has finite exponent $n$ let $m$ be an integer relatively prime to $n$ and let $\mathcal{V}_{1}$ be a variety of finite exponent $m$. Since $\mathcal{V}$ satisfies the law $X^{n}=1$ and $\mathcal{V}_{1}$ satisfies the law $X^{m}=1$ and $(m, n)=1$ it follows that their intersection satisfies the law $X=1$. Hence only trivial groups are in their intersection. But $\mathcal{V}_{1}$ is nontrivial contradicting the stated property. Therefore $\mathcal{V}$ must have infinite exponent.

\section{Extensions to Arbitrary Groups.}

As pointed out by Turner the characterization and determination of test elements in arbitrary non-free groups is much more subtle and complicated than in free groups. First we show that there can exist test elements in non-free groups. The fact that $[x, y]$ is a test word in the free group of rank two on $x, y$ followed from the following method of Nielsen: if $u, v$ are elements of the free group of rank two on $x, y$ and $[x, y]=[u, v]$ then the set $\{u, v\}$ is Nielsen equivalent to the set $\left\{x^{ \pm 1}, y^{ \pm 1}\right\}$. Exactly the same type of Nielsen transformation arguments can be applied in the free product of two cyclic groups ( not excluding finite) provided that we allow an extended Nielsen transformation which replaces a generator $x$ by $x^{d}$ where $x^{d}$ is also a generator of $\langle x\rangle$. In particular in a free product of cyclic groups with basis $x, y$ the commutator is a test element. 
Theorem 8. Let $G=\left\langle x, y: x^{p}=y^{q}=1\right\rangle \cong \mathbb{Z}_{p} \star \mathbb{Z}_{q}$ be the free product of two finite cyclic groups. Then the commutator $[x, y]$ is a test element.

Much of the development on almost primitive elements and generic elements can be translated to more general situations. Let $\mathcal{U}$ be a variety defined by the set of laws $\mathcal{V}$ and $G$ a group. Then we say that $\mathcal{U}$ is efficient for $G$ if $\mathcal{V}(H) \neq H$ for any non-trivial subgroup of $G$. Recall that an element $g \in G$ is primitive if $G=\langle g\rangle \star G_{1}$ with $G_{1} \neq G$ and $g$ of infinite order. We then get the following.

Lemma 1. Let $g$ be primitive in $G$. Then $g \notin \mathcal{V}(g)$ for any non-trivial set of laws $\mathcal{V}$ unless $\mathcal{V}(G)=G$.

Proof. If $g$ is primitive in $G$ and $H$ is any group then any map $g$ into $H$ can be extended to a homomorphism $G \rightarrow H$. Let $H$ be a $\mathcal{V}$-group. Then any $g_{1} \in \mathcal{V}(G)$ goes to the identity. Therefore $g \notin \mathcal{V}(G)$ unless $\mathcal{V}(G)=G$.

From this we can extend Theorem 1 almost exactly.

Theorem 9. Let $\mathcal{U}$ be a variety defined by the laws $\mathcal{V}$ and suppose $\mathcal{U}$ is efficient for $G$. Let $g \in \mathcal{V}(G)$. Then if $g$ is an APE in $G$ it follows that $g$ is $\mathcal{U}$-generic. Further if $G$ is Hopfian then $g$ is a test element.

Proof. The proof is almost identical to the proof of Theorem 1. Suppose $g \in \mathcal{V}(G)$. Let $K \subset G$ be a proper subgroup. If $g \notin K$ then $g \notin \mathcal{V}(K)$. If $g \in K$ then $\mathcal{V}(K) \neq K$ since $\mathcal{U}$ is efficient for $G$. Since $g$ is an APE it is primitive in $K$ and hence from Lemma $1 g \notin \mathcal{V}(K)$. Therefore $g$ is $\mathcal{U}$-generic. If $G$ is Hopfian then as before generic elements are test elements.

As an example consider the Modular group $M=\mathbb{Z}_{2} \star \mathbb{Z}_{3}$ the free product of a cyclic group of order two and a cyclic group of order three. Let $M=$ $\left\langle x, y ; x^{2}=y^{3}=1\right\rangle$ and let $g=[x, y]$. Now $[x, y]=x y x y^{2}$ so the same proof as in the free group case shows that any Nielsen transformation will map this to a cyclic rewrite up to conjugation and exponent \pm 1 . It follows than that $g$ is an APE. The abelian variety $\mathcal{A}$ is $M$-efficient so from Theorem 9 , $g$ is $\mathcal{A}$-generic. Since $M$ is Hopfian this gives another proof that $g$ is a test element.

The following straightforward propositions give some additional results.

Proposition 2. Let $F$ be a free group. $w \in F$ is a test word if and only if whenever $f: F \rightarrow F$ is an endomorphism with $f(w)=w_{1}$ with $w_{1}$ Whitehead related to $w$ then $f$ is an automorphism. 
Proof. Suppose $w \in F$ is a test word and suppose $f: F \rightarrow F$ is an endomorphism with $f(w)=w_{1}$ with $w_{1}$ Whitehead related to $w$. Then there is an automorphism $\alpha: F \rightarrow F$ with $\alpha\left(w_{1}\right)=w$. Then $f_{1}=\alpha f$ is an endomorphism of $F$ with $f_{1}(w)=w$. Since $w$ is a testword it follows that $f_{1}$ is an automorphism. Therefore $f=\alpha^{-1} f_{1}$ is also an automorphism. The converse is clear.

Proposition 3. Let $w$ be a test word in the free group $F$ and let $N$ be a normal subgroup of $F$. Suppose that whenever $w \equiv w_{1}(N)$ it follows that there is a $w_{2} \in w_{1} N$ which is Whitehead related to $w$. Let $p: F \rightarrow F / N$ be the natural projection and let $g=p(w)$. Then $g$ is a test element in $G=F / N$.

Proof. Let $\phi: G \rightarrow G$ be an endomorphism with $\phi(g)=g$. Relative to a fixed generating system of $G, \phi$ can be lifted to an endomorphism $\phi^{\star}$ of the free group $F$. Let $w_{1}=\phi^{\star}(w)$. Since $\phi(g)=g$ it follows that $p(w)=$ $p\left(w_{1}\right)$ and hence $w \equiv w_{1}(N)$. From the condition we may assume that $w$ is Whitehead related to $w_{1}$ and hence from Proposition 1 it follows that $\phi^{\star}$ is an automorphism of the free group and therefore $\phi$ is an automorphism of $G$.

\section{The Test Rank of a Group.}

If $g$ is a test element of a group $G$ then it is straightforward to see that this is equivalent to the fact that if $f(g)=\alpha(g)$ for some endomorphism $f$ of $G$ and some automorphism $\alpha$ of $G$ then $f$ must also be an automorphism. A test set in a group $G$ consists of a set of elements $\left\{g_{i}\right\}$ with the property that if $f$ is an endomorphism of $G$ and $f\left(g_{i}\right)=\alpha\left(g_{i}\right)$ for some automorphism $\alpha$ of $G$ and for all $i$ then $f$ must also be an automorphism. Any set of generators for $G$ is a test set and if $G$ posses a test element then this is a singleton test set. The test rank of a group is the minimal size of a test set. Clearly the test rank of any finitely generated group is finite and bounded above by the rank and below by 1 . Further the test rank of any free group of finite rank is 1 since these contain test elements. For a free abelian group of rank $n$ the test rank is precisely $n$.

Lemma 2. If $G=\mathbb{Z}^{n}, n \geq 1$ is a free abelian group of rank $n$ then its test rank is $n$.

Proof. Let $G=\mathbb{Z} \times \mathbb{Z}$ be a free abelian group of rank 2 . We show that $G$ contains no test element. The proof in the general case that a set of $k$ elements in a free abelian group of rank $n$ with $k<n$ cannot be a test set is analogous. 
Let $x, y$ be a basis for $G$. We will write the group additively. Suppose $g=m x+n y$ with $m, n$ integers is a test element. We will show that there exists a non-invertible endomorphism of $G$ which fixes $g$. Any mapping

$$
\begin{aligned}
& x \rightarrow a x+b y \\
& y \rightarrow c x+d y
\end{aligned}
$$

determines an endomorphism of $G$ to $G$. This homomorphism will be invertible and hence an automorphsim only if $a d-b c= \pm 1$. Suppose under this homomorphism $g \rightarrow g$. We thus have

$$
m x+n y \rightarrow m(a x+b y)+n(c x+d y)=g=m x+n y .
$$

Considering $a, b, c, d$ as integral unknowns we are then led to the sytem of two equations in four unknowns

$$
\begin{aligned}
& m a+n c=m \\
& m b+n d=n .
\end{aligned}
$$

This has infinitely many integral solution with $c$ dependent on $a$ and $b$ dependent on $d$. Choosing one such solution such that $a d-b c \neq \pm 1$ gives the desired homomorphism.

Thus free abelian groups have maximal test rank while free groups of finite rank have minimal test rank. For given integers $n$ and $k$ with $k<n$ there always exist groups of rank $n$ and test rank $k$.

Lemma 3. Given integers $n$ and $k$ with $k<n$ there exist a group of rank $n$ and test rank $k$.

Proof. Let $G_{m}$ stand for a free abelian group of rank $m$ and $F_{d}$ stand for a free group of rank $d$. Then the group $G=F_{d} \times G_{m}$ has rank $m+d$ and test rank $m+1$. Given arbitrary $n$ and $k<n$ choose $m, d$ so that $m+1=k$ and $m+d=n$. The group $G$ then has the desired property.

We close with two questions on test rank.

(1) Given a finite presentation for a group $G$ and given knowledge of the rank can one determine the test rank?

(2) Can one give an example of a group $G$ with rank $n$ and test rank $1<k<n$ other than those of the type in the proof of Lemma 3 - that is not of the form $F_{d} \times G_{m}$. 


\section{References}

[B-B-O] A.M. Brunner, R.G. Burns and S. Oates-Williams, On almost primitive elements of free groups with an application to Fuchsian groups, Can. J. Math., 45 (1993), $225-254$.

[C] L.P. Comerford, Generic elements of free groups, Archiv der Math, to appear.

[C-E] L.P. Comerford and C.C. Edmonds, Products of commutators and products of squares in a free group, Int. J. of Algebra and Computation, 4 (1994), 469-480.

[Do] A. Dold, Nullhomologous words in free groups which are not nullhomologous in any proper subgroup, Arch. Math., 50 (1988), 564-569.

[D] V.G. Durnev, The Mal'cev-Nielsen equation in a free metabelian group of rank two, Math. Notes, 64 (1989), 927-929.

[F-R-S] B. Fine, G. Rosenberger and M. Stille, Nielsen Transformations and Applications: A Survey, Groups Korea, 1994, Kim/Johnson Eds., DeGruyter, (1995), 69-105.

[G-S] N. Gupta and V. Shpilrain, Nielsen's commutator test for two-generator groups, Arch. Math., 44 (1985), 1-14.

[H-P] P. Hill and S. Pride, Commutators, generators and conjugacy equations in groups, Math Proc. Camb. Phil. Soc., 114 (1993), 295-301.

[I] N. Isermann, Generische Elemente und Testelemente in freien Gruppen, Diplomarbeit - Universitat Dortmund, 1996.

[K-R] R.N. Kalia and G.Rosenberger, Automorphisms of the Fuchsian groups of type (0, 2, 2, 2, q:0), Comm. in Alg., 6(11) (1978), 115-129.

[M-P] J. McCool and A. Pietrowski, On free products with amalgamation of two infinite cyclic groups, J. of Alg., 18 (1971), 377-383.

[Ne] H. Neumann, Varieties of Groups, Springer-Verlag, 1967.

[N] J. Nielsen, Die Automorphisem der algemeinen unendlichen Gruppe mit zwei Erzeugenden, Math. Ann., 78 (1918), 385-397.

[R1] G. Rosenberger, Uber Darstellungen von Elementen und Untergruppen in freien Produkten, Proc. of Groups Korea 1983: Springer Lecture Notes in Math., 1098 (1984), 142-160

[R2] - Alternierende produkte in freien Gruppen, Pac. J. Math., 78 (1978), 243-250.

[R3] _ Minimal generating systems for plane discontinuous groups and an equation in free groups, Proc. of Groups Korea 1988: Springer Lecture Notes in Math., (1989), 170-186.

[S1] V. Shpilrain, Test elements for endomorphisms of free groups and algebras, preprint.

[S2] _ Recognizing automorphisms of the free groups, Arch. Math., 62 (1994), 385-392.

[St] J. Stallings, Problems about free quotients of groups, preprint.

[T] E.C. Turner, Test words for automorphisms of the free groups, J. London Math. Soc., to appear.

[Z1] H. Zieschang, Alternierende produkte in freien Gruppen, Abh. Math. Sem. Univ. Hamburg, 27 (1964), 12-31. 
[Z2] _ Automorphismen ebener discontinuerlicher Gruppen, Math. Ann., 166 (1966), 148-167.

[Z3] _ Uber die Nielsensche Kurzungsmethode in freien Produkten mit Amalgam, Invent. Math., 10 (1970), 4-37.

Received March 7, 1997 and revised May 18, 1998.

FAIRFIELD UNIVERSity

FAIRFIELD, CT 06430

E-mail address: fine@fair1.fairfield.edu

Fachbereich Mathematik Universitat

DORTMUND

Federal Republic of Germany

St Joseph UnIVERsity

Philadelphia, PA 19131

FaChbereich Mathematik Universitat

DORTMUND

Federal Republic of Germany 